\title{
Current oscillations in Vanadium Dioxide: evidence for electrically triggered percolation avalanches
}

\author{
Tom Driscoll, ${ }^{1,2, a)}$ Jack Quinn, ${ }^{2}$ Giwan Seo, ${ }^{3}$ Yong-Wook Lee, ${ }^{4}$ Hyun-Tak Kim, ${ }^{3,5}$ David R. Smith, ${ }^{1}$ Massimiliano \\ Di Ventra, ${ }^{2}$ and Dimitri N. Basov ${ }^{2}$ \\ ${ }^{1)}$ Center for Metamaterials and Integrated Plasmonics, Pratt School of Engineering, Duke University. Durham, NC, \\ 27708, USA \\ 2) Physics Department, University of California, San Diego, La Jolla, California 92093, \\ USA \\ ${ }^{3)}$ School of Advanced Device Technology, University of Science and Technology (UST). Daejon, 305-350, \\ Korea. \\ ${ }^{4)}$ School of Electrical Engineering, Pukyong National University, Busan 608-737, \\ South Korea. \\ ${ }^{5)}$ Creative research center of Metal-insulator transition, ETRI, Daejon 305-700, \\ South Korea.
}

(Dated: 25 October 2018)

In this work, we experimentally and theoretically explore voltage controlled oscillations occurring in microbeams of vanadium dioxide. These oscillations are a result of the reversible insulator to metal phase transition in vanadium dioxide. Examining the structure of the observed oscillations in detail, we propose a modified percolative-avalanche model which allows for voltage-triggering. This model captures the periodicity and waveshape of the oscillations as well as several other key features. Importantly, our modeling shows that while temperature plays a critical role in the vanadium dioxide phase transition, electrically induced heating cannot act as the primary instigator of the oscillations in this configuration. This realization leads us to identify electric field as the most likely candidate for driving the phase transition.

\section{INTRODUCTION}

Vanadium Dioxide $\left(\mathrm{VO}_{2}\right)$ has been a material of prolonged scientific interest, due to the plethora of unusual properties associated with the Insulator to Metal phase Transition (IMT) occurring just above room temperature ${ }^{1}$. The large conductivity change ratio, combined with an accessible transition temperature and rich correlated-electron physics ${ }^{2}$ has made this an attractive compound for many researchers. Much attention has historically revolved around controversy over the driving physics of the phase transition; particularly whether it is a Mott transition ${ }^{5-7]}$ or Pierels transition 289 . However, also of interest is the ability of the IMT to happen on ultrafast (100fs) timescales 10 , and the wide range of stimuli which can trigger it ${ }^{11 / 12}$. Along these lines, recent interest has also shifted from purely academic to industrial as well; following proposed applications ranging from optical devices ${ }^{13}$ and hybrid-metamaterials ${ }^{14}$ to electronic components $\frac{17 \mid 18}{1}$ and data storage $\frac{1920}{}$. With this rise of potential applications comes opportunities for new avenues of research and development, but also new challenges to satisfy the durability and flexibility that real-world devices demand ${ }^{21}$. Understanding the role of temperature and structural transitions in various $\mathrm{VO}_{2}$ phenomenon is key to pushing towards potential of applications.

In this manuscript we take an interest in the recently reported ${ }^{22 \mid 23}$ phenomenon of self-sustaining oscillations

a) Tom.Driscoll@Duke.edu in $\mathrm{VO}_{2}$ bridges. The widespread prevalence of voltage controlled oscillators in electronics makes this phenomenon an enticing candidate for devices. It is fairly well accepted that these oscillations represent a triggering of the Insulator-to-Metal Transition (IMT), followed by a reseting Metal-to-Insulator Transition (MIT). However, despite headway on controlling such oscillations in $\mathrm{VO}_{2} 2324$, there is still debate over whether the underlying driving mechanism is thermal or electrostatic. In literature, $\mathrm{VO}_{2}$ is most often thermally triggered, and yet these oscillations appear to respond to foremost to applied voltage across the device. The unavoidable presence of joule-heating currents through the 2-terminal device during operation, coupled with the observed sensitivity of the oscillations to device temperature ${ }^{2125}$, make for a contentious situation.

In our investigation we first experimentally reproduce the oscillations discovered by the authors of Ref22. The use of a high-performance oscilloscope in our experiment gives us access to very fine time-resolution data which is useful in our modeling. The details and data of our experiment are reported in Section II. Following this, we develop a model which replicates and explains the observed waveshape in terms of electricallytriggered domains. Our model, reported in Section III, describes a network of electrically and/or thermally triggered grains. This model is inspired by several previously proposed models 2627 . While these previous models also predict avalanche-like transitions under the right conditions ${ }^{26}$, our our model expands on this framework to track time-dependent effects and allow possibility of a voltage-triggered phase-transition. Alongside voltage triggering, we investigate the role of temperature in the 


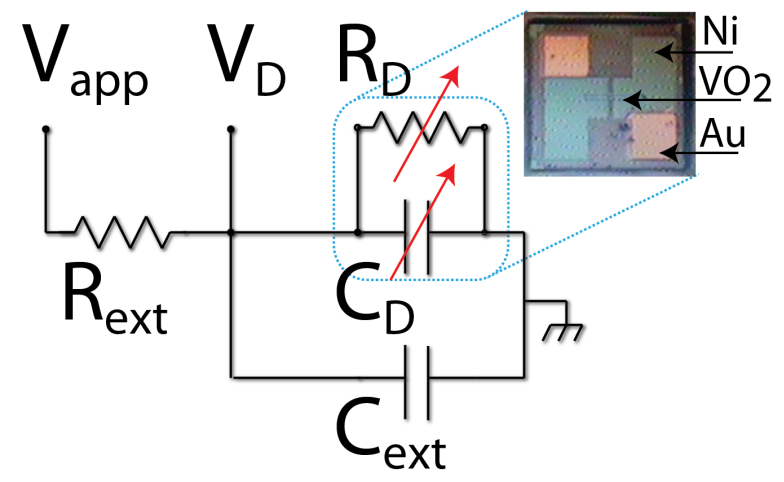

FIG. 1. Schematic of the circuit diagram used to reproduce oscillations, depicting the $\mathrm{VO}_{2}$ device as a variable resistance and capacitance. Inset shows optical photograph of a sample device. The $\mathrm{Al}_{2} \mathrm{O}_{3}$ substrate is $330 \mu \mathrm{m}$ thick, and is mounted on a glass cover slip. All experiments are performed at room temperature.

oscillations, and importantly, we find while a voltagedriven picture replicates experimental data - thermal heating alone is quantitatively and qualitatively unable to explain the structure of the observed oscillations. Nevertheless, device temperature does affect oscillations, and thermal co-factors to the voltage triggering are needed to reproduce aspects of the data. In Section $[\mathrm{IV}$ we discuss how the percolative transition of $\mathrm{VO}_{2}$ affects the shape of the MIT, and what this means regarding effective medium within the phase-coexistence region. In Section $\nabla$ we conclude the manuscript with an overview of our results, and an outlook on possible directions for $\mathrm{VO}_{2}$ research and application.

\section{VOLTAGE CONTROLLED OSCILLATIONS.}

Our investigation begins with replicating $\mathrm{VO}_{2}$ oscillations using the procedure reported by Kim et.al2224. A device consisting of a $10 \mu \mathrm{m} \times 10 \mu \mathrm{m} \mathrm{VO}_{2}$ bridge between two large $(\sim 400 \mu \mathrm{m})$ metal $(\mathrm{Ni}: \mathrm{Au})$ electrodes (Figure 1 . inset $)$ is hooked in series with a limiting resistor $\left(\mathrm{R}_{e x t}\right)$ and voltage source $\left(\mathrm{V}_{\text {app }}\right)$. This setup is shown schematically in Figure 1. Although we do not intentionally add external capacitance, the presence of such $\mathrm{C}_{e x t}$ in instruments and cables is unavoidable - and should be included in the effective circuit. The applied voltage $\mathrm{V}_{a p p}$ is a transient square pulse (between $1 \mu$ s to $1 \mathrm{~ms}$ ) from a Agilent function generator (model 33120A) riding on top of a constant bias voltage $\left(\mathrm{V}_{\text {bias }}=12 \mathrm{~V}\right)$. This is shown as the black trace in Figure 2, giving a peak applied voltage of $22 \mathrm{~V}$. The voltage across the device $\left(\mathrm{V}_{D}\right.$, as shown by the blue trace in Figure 2 is monitored with a LeCroy (model wavepro 7-zi) oscilloscope, which allows for high time-resolution $(40 \mathrm{GS} / \mathrm{s})$ sampling resolution even over millisecond-long pulses.

In this configuration, the $\mathrm{VO}_{2}$ device functions essentially as a capacitor with a variable internal shunt

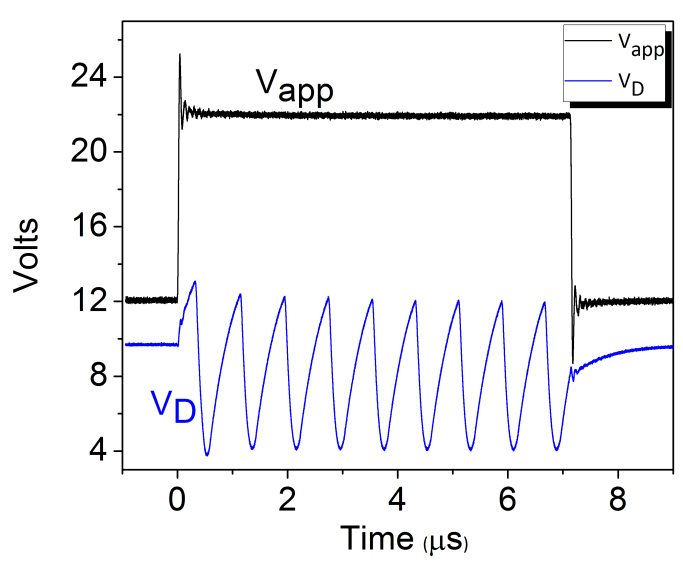

FIG. 2. Applied voltage and voltage across the device as a function of time.

resistance $\mathrm{R}_{D}$. The capacitance $\mathrm{C}_{D}$ is primarily fixed by device geometry, although variations of the dielectric constant of $\mathrm{VO}_{2}$ throughout the phase transition (such as have been shown in the context of memorycapacitanc ${ }^{15228}$ and $\mathrm{VO}_{2}$ hybrid-metamaterials ${ }^{14}$ ) may have small effects - and we discuss this later in Section IV B The pre-pulse steady-state starting voltage is $\mathrm{V}_{D}=\mathrm{V}_{\text {bias }} \mathrm{R}_{D} /\left(\mathrm{R}_{D}+\mathrm{R}_{\text {ext }}\right)$. At the start of the pulse $(\mathrm{t}=0), \mathrm{V}_{D}$ increases, following a canonical ResistanceCapacitance $(\mathrm{RC})$ charging curve. Once $\mathrm{V}_{D}$ surpasses a threshold voltage (which we will call $\mathrm{V}_{D: I M T}$ ), it transitions sharply from increasing $\mathrm{V}_{D}$ to decreasing. We assign this change to an IMT event occurring in the $\mathrm{VO}_{2}$, which effectively lowers the internal shunt resistance $\mathrm{R}_{D}$ of the capacitor. With lower internal resistance, the capacitor undergoes rapid discharge and $\mathrm{V}_{D}$ plummets. This discharge continues until $\mathrm{V}_{D}$ reaches a lower threshold voltage $\left(\mathrm{V}_{D: M I T}\right.$ at which a second event - which we similarly assign to a a Metal-to-InsulatorTransition (MIT) - restores the high internal device resistance. The process reverses and this sequence of events repeats; alternating charging and discharging between IMT and MIT events with a fairly stable periodicity 24 .

\section{GRAIN-SCALE MODEL OF OSCILLATIONS.}

Our hope is that by developing a model for these observed oscillations, we may gain insight into the driving mechanism behind them. We start with a $2 \mathrm{D}$ network of square $\mathrm{VO}_{2}$ grains of differing size, each with total resistivity dependent on its size and its state (metal or insulator ${ }^{29 / 30}$. The granularity of polycrystaline $\mathrm{VO}_{2}$ is well documented $\sqrt{31 / 32}$, although the size of grains may vary considerably from one $\mathrm{VO}_{2}$ preparation to another. There is also evidence to suggest the percolation length scales for the IMT may not always coincide with the crystal granularity 33 35. A cartoon illustrating our model ar- 


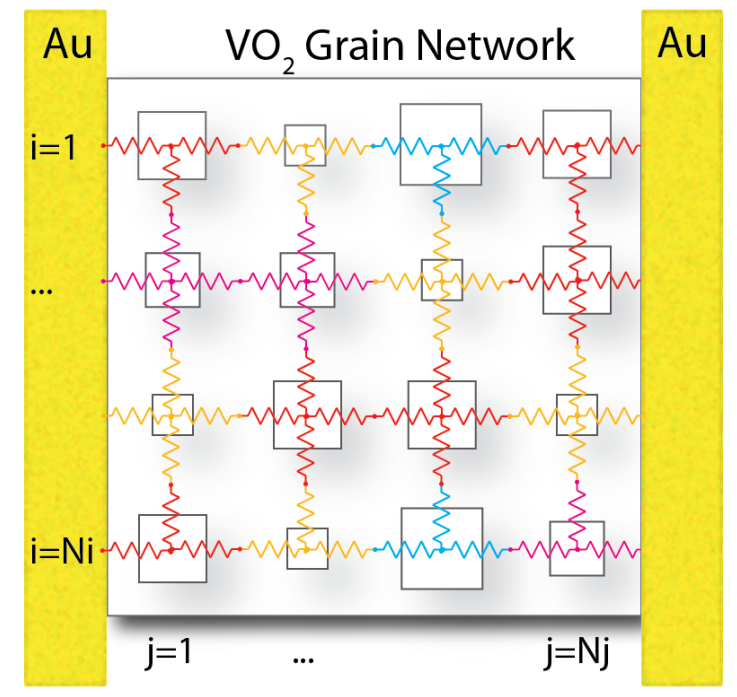

FIG. 3. Cartoon of the grains resistor-network. Left and right sides are the beginning of the nickel electrodes. In the cartoon, the size of squares represent a distribution of grain sizes and resistor colors serve to highlight the corresponding different resistance.

rangement is shown in Figure 3. The network consists of $\mathrm{N}_{i} \mathrm{xN}_{j}$ grains, and in our model we restrict our investigation to a 50x50 network array to keep computation time manageable.

This grain network is placed in an external circuit containing resistance $\mathrm{R}_{\text {ext }}$ and capacitance $\mathrm{C}_{e x t}$, and driven by $\mathrm{V}_{a p p}$, as shown in Figure 1 . The circuit differential equation (Equation 1), is solved via Runge-Kutta timestepping.

$$
\dot{V}_{D}(t)=\frac{1}{\left(C_{D}+C_{e x t}\right)}\left(\frac{V_{a p p}-V_{D}}{\left(R_{e x t}\right)}-\frac{V_{D}}{\left(R_{D}\right)}-\dot{C}_{D} V_{D}\right)
$$

At each time-step, we solve for the internal state of the grain network. This includes solving a Kirchoff network problem ${ }^{36}$ for the voltage across each grain and the Thevenin effective circuit resistance $\mathrm{R}_{D}$. The other $\mathrm{VO}_{2}$ effective circuit parameter $\mathrm{C}_{D}$ is found by a differential capacitance equation (Equation 2) which can be evaluated via a self-consistent Bruggeman effective medium formulation (Equations 34 )

$$
\begin{aligned}
C_{D}(t) & =C_{0}+\eta \frac{\epsilon_{D}}{\epsilon_{0}} \\
0 & =f \frac{\epsilon_{m}-\epsilon_{D}}{\epsilon_{m}+2 \epsilon_{D}}-(f-1) \frac{\epsilon_{i}-\epsilon_{D}}{\epsilon_{i}+2 \epsilon_{D}} \\
f & =\frac{\sum^{i j} X^{i j}}{N_{i} N_{j}} .
\end{aligned}
$$

Superscript $i$ and $j$ are row and column indices for the grains (running to $\mathrm{N}_{i}$ and $\mathrm{N}_{j}$ total). The binary matrix $\mathrm{X}^{i j}=1$ if the grain $i, j$ is metal and 0 if insulator. $\eta$ is a capacitive fractional-fields factor (the proportion of the device capacitance which involves the $\mathrm{VO}_{2}$ dielectric), which is found via finite element simulation using the COMSOL commercial code package. $\mathrm{C}_{0}$ is a geometrical capacitance which is determined empirically, fitting 1/RC to the capacitive charging curve. External circuit parameters such as $\mathrm{R}_{e x t}$ and $\mathrm{V}_{a p p}$ are taken directly from experimental values. The extrema values for $\mathrm{R}_{D}$ (metal) and $\mathrm{R}_{D}$ (insulator) are taken from temperature data.

\section{A. Thermal triggering}

The temperature driven IMT-MIT has been investigated in great detail and we ground our model using experimental data giving resistance as a function of temperature $R(T)$ through the phase transition. This data is shown in Figure 4 a, and displays the characteristic sharp change in resistivity around $345 \mathrm{~K}$.

Then, in a procedure similar to previous works ${ }^{626}$, we assume each grain will undergo an IMT in response a "high-threshold" temperature $\mathrm{T}_{I M T}^{i j}$, and a MIT at a low-threshold $\mathrm{T}_{M I T}^{i j}$.

$$
\begin{aligned}
R^{i j} & =R_{m e t} \quad \text { if }\left(T^{i j}>T_{I M T}^{i j}\right) \\
& =R_{\text {ins }} \quad \text { if }\left(T^{i j}<T_{M I T}^{i j}\right)
\end{aligned}
$$

We assign a stochastic distribution to the values of $T_{I M T}^{i j}$ and $T_{M I T}^{i j}$ throughout the network, following the Gaussian form

$$
\begin{gathered}
P\left(T_{I M T}^{i j}\right)=e^{-\frac{\left(T_{I M T}^{i j}-T_{0_{I M T}}\right)}{2 \sigma_{I M T}^{2}}} \\
P\left(T_{M I T}^{i j}\right)=e^{-\frac{\left(T_{M I T}^{i j}-T_{0_{M I T}}\right)}{2 \sigma_{M I T}^{2}}} .
\end{gathered}
$$

The values of $\mathrm{T}_{0}$ and $\sigma^{2}$ are fit to the experimental $\mathrm{R}(\mathrm{T})$ data shown in Figure 4 a. From this, we find a variance of $T_{0}=340^{\circ} \mathrm{K}$ and $\sigma_{T}^{2}=.1^{*} T_{0}$ reproduces the shape of the IMT fairly well. This fit gives us confidence that we understand the thermal response of $\mathrm{VO}_{2}$, even though our thermal model is simple compared to some previous treatments.

Using this temperature-only-triggered model, we attempt to reproduce the oscillations shown in Figure 2. Our model tracks the power dissipated in each grain and employs a finite-element method to solve for the grain and substrate temperatures as a function of time. Material thermal parameters are taken from literature, and the enthalpy of phase-transition for $\mathrm{VO}_{2}$ is included. Figure $4 \mathrm{~b}$ re-plots our experimental $V_{D}$ oscillations in blue, and the model results in green. The result is striking: although we can track $V_{D}$ for a short while, we are unable to produce any oscillatory phenomena.

Generalizing the behavior that prohibits oscillations, thermal-initiated IMTs exhibit a runaway behavior rather than the self-stabilizing oscillatory nature seen in Figure 2. This is apparent in the average $\mathrm{VO}_{2}$ grain 


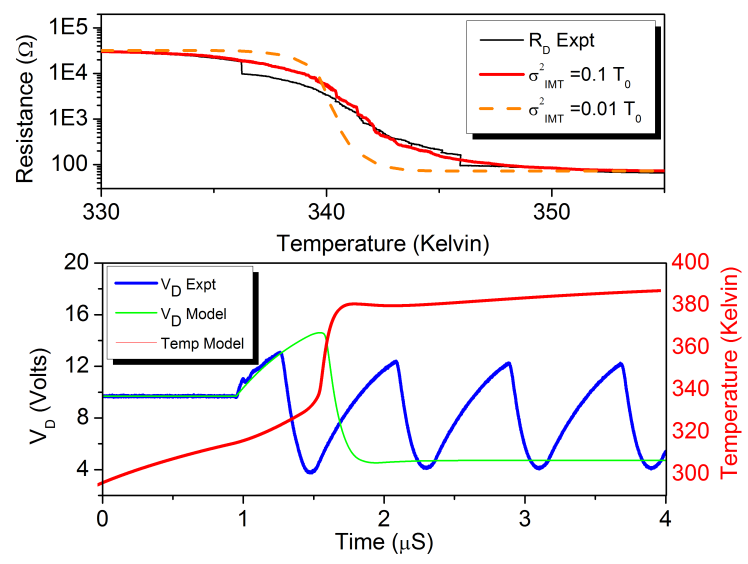

FIG. 4. a) Experimental resistance of our device as a function of temperature $\mathrm{R}_{D}(\mathrm{~T})$ (black). $\mathrm{R}_{D}(\mathrm{~T})$ results from our model are overlaid for a best-fit value of variance: $\sigma_{I M T}^{2}=0.1 * T_{0}$ (see Equation 7), which replicates the observed temperaturedriven IMT fairly well. Also shown is a poor-fit result for $\sigma_{I M T}^{2}=0.01 * T_{0}$, given to show the effect of this parameter. b) Attempt to replicate oscillations using thermal-only triggering, with values of $P\left(T_{I M T}^{i j}\right)$ from above. We plot $\mathrm{V}_{D}$ from our model (green) overlaid on experimental $V_{D}$ (blue). We observe no oscillations, only a single IMT event. We also plot the average $\mathrm{VO}_{2}$ temperature $\mathrm{T}_{D}$ from our model, illustrating a runaway heating behavior that precludes oscillations.

temperature plot (red) of $4 \mathrm{p}$. Note that although individual different grains may attain different temperatures over the course of oscillations, such gradients equalize quickly within the network. Average temperature remains a fairly accurate and easily visualized metric of the $\mathrm{VO}_{2}$ oscillation thermodynamics. As the thermallytriggered IMT occurs, $\mathrm{VO}_{2}$ temperature skyrockets even while $\mathrm{V}_{D}$ discharges. Once the temperature of the discharged device has surpassed $T_{0_{I M T}}$, there is no way for it to cool thereafter. Power dissipation and temperature both settle towards steady-state with the device firmly in the metallic state. The causes of this process will become more apparent as we discuss thermal dynamics in Section IIID.

\section{B. Electrical triggering}

With the failure of a temperature-only-triggering model to produce oscillations, and following insights from previous work on voltage-induced effects in $\mathrm{VO}_{2}, 2226 \sqrt{3738}$ we now introduce an electric-field driven transition. To do this, we also assign a voltage drop at which each grain undergoes phase transition $\mathrm{V}_{I M T}^{i j}$ and $\mathrm{V}_{M I T}^{i j}$. We again use random values from a normal dis-

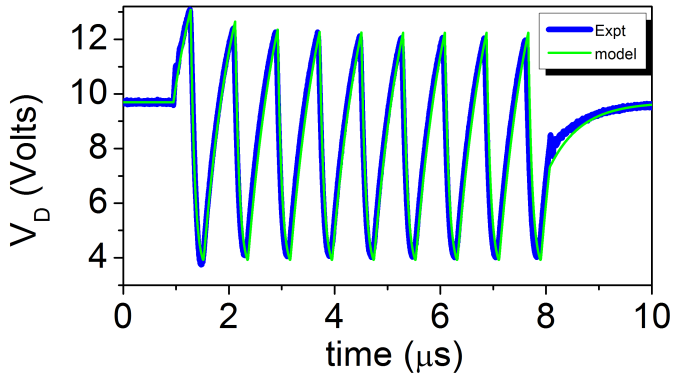

FIG. 5. The experimental data from Figure 2 replotted (blue) with numerical results from our model overlaid (green). This electrical-and-thermal triggering model replicates the experimental data quite well, tracking the oscillation periodicity and producing similar transitions at both $\mathrm{V}_{D: I M T}$ and $\mathrm{V}_{D: M I T}$.)

tribution as we did for $\mathrm{T}_{I M T}^{i j}$ and $\mathrm{T}_{M I T}^{i j}$.

$$
\begin{gathered}
P\left(V_{I M T}^{i j}\right)=e^{-\frac{\left(V_{I M T}^{i j}-V_{0_{I M T}}\right)}{2 \sigma_{I M T}^{2}}} \\
P\left(V_{M I T}^{i j}\right)=e^{-\frac{\left(V_{M I T}^{i j}-V_{0_{M I T}}\right)}{2 \sigma_{M I T}^{2}}} .
\end{gathered}
$$

We lack direct data to which to fit these distributions (as we did in Figure 4a). Thus, we retain the same value for the variance found in above, $\sigma_{V}^{2}=0.1 * \mathrm{~V}_{0}$. From an energetics perspective, this makes a great deal of sense - both distributions are surely tied to the same underlying Mott-physics. $\mathrm{V}_{0}$ remains a fitting parameter in our model.

As we have not removed possible thermal triggering, the conditions for grain transition can now be stated as:

$$
\begin{aligned}
R^{i j} & =R_{m e t} \quad \text { if }\left(\left(V^{i j}>V_{I M T}^{i j}\right) \text { OR }\left(T^{i j}>T_{I M T}\right)\right) \\
& =R_{\text {ins }} \quad \text { if }\left(\left(V^{i j}<V_{M I T}^{i j}\right) \text { AND }\left(T^{i j}<T_{M I T}\right)\right)
\end{aligned}
$$

This combined triggering criterion reproduces both the waveshape and periodicity of the experimentally observed oscillations quite well. In Figure 5, we re-plot the experimental data from Figure 2 in blue, and numerical results from our model are shown overlaid in green.

\section{Voltage-temperature dependence}

Although section III B demonstrated voltage is the primary trigger, device temperature still plays a role in oscillations. There is a known dependence of oscillation amplitude on device temperature 25 . If we look carefully at the data in Figure 5 , we notice a subtle decay envelope to the amplitude of the $\mathrm{V}_{I M T}$ oscillation peaks. We believe this envelope is caused by a thermalization of the device on a multi-oscillation timescale. To accommodate 


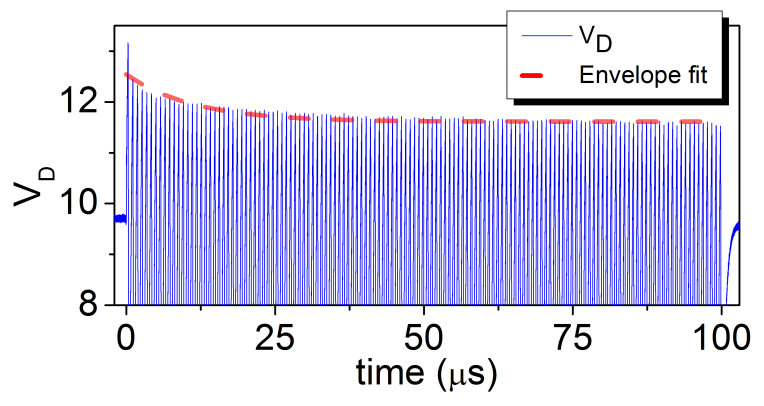

FIG. 6. Experimental data (blue) for oscillations over a long $100 \mu \mathrm{s} \mathrm{V}_{\text {app }}$ pulse. We observe a clear decay envelope to the amplitude of $\mathrm{V}_{I M T}$ in the oscillations. For clarity, the bottom half of the oscillations are omitted as we observe no enveloping here. To show this decay is a thermalization envelope caused by device heating, we also plot the temperature dependent $\mathrm{V}_{0_{I M T}}(T)$ from equation 13 (red).

this, we include a temperature-dependence to the IMT transition voltage as

$$
V_{I M T}^{i j}(T) \rightarrow\left(\kappa\left(T-T_{0}\right)+1\right) V_{I M T}^{i j}
$$

where $\mathrm{T}_{0}=295^{\circ} \mathrm{K} . \kappa$ is a liner temperature coefficient, the fitting of which we discuss below. Without including this voltage-temperature interplay, our model quickly loses sync with the experimental data over the course of several oscillation periods. This thermalization envelope is most clearly observed over a long pluse, and experimental data (solid blue) for a $100 \mu \mathrm{s}$ pulse is shown in Figure 6. For clarity in this figure, the bottom half of the oscillations is omitted from view (interestingly, we observe no envelope of $\left.\mathrm{V}_{M I T}\right)$.

Using the data from Figure 6 combined with our thermal-finite element model, we can fit a value for $\kappa$. Overlaid on the experimental data (dashed line) is this fit, which gives $\kappa=-8.3 \times 10^{-3}$. This value of $\kappa$ is used in our oscillation model.

Explanation of this a long time-scale thermalization is straightforward. Although locally the $\mathrm{VO}_{2}$ film may heat or cool quite quickly in response to current through its volume, the $500 \mu \mathrm{m}$ thick sapphire substrate is comparatively massive. The large thermal inertia of the substrate smooths out oscillatory heating away from the film; and when heated only from the top the substrate can require tens of micro-seconds to reach a steady-state temperature gradient.

\section{Temperature dynamics}

In the above Section $\amalg I \mathrm{C}$ we have discussed the critical interplay between temperature and voltage-triggering, showing how a long multi-oscillation timescale thermalization envelopes the oscillation amplitude. In this section, we look closer at the temperature evolution on the timescale of the oscillation period.

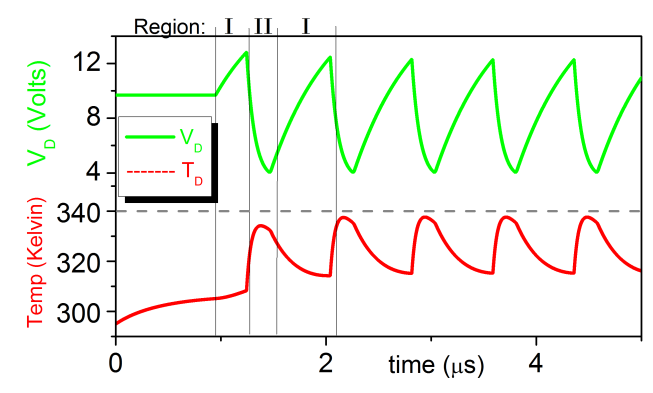

FIG. 7. Model results of temperature dynamics during $\mathrm{V}_{D}$ oscillations. The horizontal dashed line at $\mathrm{T}_{I M T}=340^{\circ} \mathrm{K}$ shows mean transition temperature, and demonstrates that the average temperature reached during oscillations falls short of what's needed to drive the IMT transition. We also divide the oscillation into insulating/charging regions(I) and metallic/discharge regions(II). This draws attention to the sharp increase in temperature which occurs only after the $\mathrm{V}_{I M T}$ trigger, qualitatively discrediting a thermal-driving picture for oscillations.

To begin, in Figure 7 we plot the average grain temperature (red) during oscillations along with $V_{D}$ (green) - both from our model. Looking at Figure 7 quantitatively, we notice that the average temperature reached is not sufficient to trigger oscillations. Although the peak average temperature during the discharge cycle of the oscillation comes close to reaching $\mathrm{T}_{I M T}$, a thermal-driving event would have to occur at-or-before the peak in $\mathrm{V}_{D}$. The model results indicate that the device is well below $\mathrm{T}_{I M T}$ when $\mathrm{V}_{D: I M T}$ is reached. The temperature range in Figure 7 agrees fairly well with previous numerical work on the subject ${ }^{39}$, and our own investigations using commercial finite element package COMSOL. Compared to COMSOL, our home-grown finite-element code over-estimates temperatures reached - perhaps due to difficulty in modeling all of the spatially massive substrate (this is not an issue for commercial packages such as COMSOL). However, commercial codes cannot be run from within Runge-Kutta time-stepping of equation 1, and our code thus critically allows us to calculate temperature dynamics during oscillations.

Although a quantitative argument for non-thermal triggering seems compelling, we are acutely aware that precisely solving for temperature can be difficult in such nanoscale systems. Material properties can differ from published bulk values, and interface effects can dominate transport and heating 40 . As we look closely though, the power dissipation in the device also appears qualitatively unfit to explain the oscillations. A thermally-driven transition would have to follow the logical sequence:

I The insulating device heats with applied $\mathrm{V}_{D}$ until it reaches $\mathrm{T}_{I M T}$, where it undergoes an IMT (becoming metallic).

II The metallic device discharges its stored capacitive energy through the its own volume - cooling as it 
discharges - until it reaches $\mathrm{T}_{M I T}$ where it undergoes MIT (becoming insulating).

\section{The process repeats.}

However, Figure 7 illustrates that region II which occurs after $\mathrm{V}_{D: I M T}$ is a region of maximum power dissipation; a region of heating not cooling. A simple ohms-law argument explains: Just before and just after the IMT, $\mathrm{V}_{D}$ is approximately $\mathrm{V}_{D: I M T}$. However, the resistance $\mathrm{R}_{D}$ has changed by a factor of 10 , and thus the power dissipated $\left(\mathrm{P}=\mathrm{V}_{D: I M T}^{2} / \mathrm{R}_{D}\right)$ is substantially greater during region II than during region I. Thus, we come to the conclusion that a purely-thermal explanation for the oscillations is qualitatively as well as quantitatively mismatched to experimental data.

Summarizing Section III, we have identified voltage as a key player in triggering observed oscillations on the grounds of several thermal arguments. One interesting question then is whether electrostatic voltage may also trigger the also IMT in a current-free (ie. FET) configuration. The joule-heating present in our two-terminal device complicates matters, in light of the voltage/temperature interplay identified in Section III C. Previous work has suggested such electrostatic switching can exist, $\stackrel{6 / 41 \mid 42}{6}$ although these early results await further confirmation. Exploring the phase-space defined by the interplay of temperature, electrostatic field, and current in these $\mathrm{VO}_{2}$ oscillations may reveal information about the correlated electron dynamics and energy scales associated with the Mott-transition.

\section{PERCOLATION}

In this section, we go into further detail on the mechanisms of the IMT and MIT transitions. Polycrystalline $\mathrm{VO}_{2}$ is known to exhibit percolative behavior during phase transition 226/34/43, and this has interesting effects on a voltage-triggered transition. Using the model from Section III which accurately predicts the observed electrical oscillations, we attempt to gain insight on several of the internal processes during oscillatory events.

\section{A. Percolative avalanche driven oscillations}

In several previous works ${ }^{26127}$, avalanche-like MIT and IMT transitions have been observed under the right conditions. The immediacy of the observed change from charging to discharging in our oscillations leads us to suspect similar avalanche behavior in our electrically-driven device. Examining the details of our model, we see that the voltage drop across any grain in the network (see Figure 3 is proportional to the resistance of the grain. During the charging cycle of the waveform voltage across the entire device $\left(\mathrm{V}_{D}\right)$ increases, and $\mathrm{V}^{i j}$ across each grain does as well. This charging continues until one "unlucky" grain hits its $\mathrm{V}_{I M T}^{i j}$ first. Because the grains receive a stochastic distribution for $\mathrm{V}_{I M T}^{i j}$ (see Equation 9), this can be a random grain anywhere in the network. In experiments, it is seen that the phase-transition is often seeded at particular places such as defects or boundaries.

The unlucky grain that first hits its IMT trigger condition undergoes an IMT. Once this grain becomes metallic, it supports a lower voltage drop $\left(\mathrm{R}_{\text {ins }} / \mathrm{R}_{\text {met }} \approx 20\right)$ - which shifts much of its voltage burden to neighboring grains. The neighboring grains in turn become increasingly likely to undergo their own IMT events. The IMT spreads across the entire sample in an avalanchelike manner. This process is depicted in Figure 8 a for a network of 50x50 grains. The upper sequence of black and white frames shows whether each grain is insulating (white) or metallic (black). The lower color frames depict the voltage drop across each grain. The neighborneighbor grain interaction, mediated by voltage drop is quite evident.

As is common in percolative systems, the Thevenin resistance $\mathrm{R}_{D}$ of the network is quite sensitive to the spatial distribution of triggered grains. The device resistivity $\mathrm{R}_{D}$ is shown above each frame in the sequence of Figure 8, and we see the largest drop occurs in frames \#6 and 7 , where the percolation path is completed from left to right. Once a conducting path forms $R_{D}$ plummets, and $\mathrm{V}_{D}$ begins to drop as the device discharges.

During discharge, there comes a point where $\mathrm{V}_{D}$ drops far enough that a similar process happens in reverse. This MIT is depicted in Figure 8b. However, the MIT process is not exactly the reverse of the IMT. The equations governing resistors in parallel tend to "favor" low-resistances in the following manner: Decreasing the value of one resistor (in a parallel network) lowers the Thevenin resistance significantly, but raising the value of a single resistor has only a little effect on the Thevenin resistance. For this reason, the avalanche-like behavior observed in Figure $8 \mathrm{a}$ is not seen in Figure $8 \mathrm{~b}$. Instead, the process much more closely resembles random percolation, with only moderate neighbor-neighbor interaction. This difference in the mechanisms between IMT and MIT may explain the difference in sharpness of the transitions at $\mathrm{V}_{D_{I M T}}$ and $\mathrm{V}_{D_{I M T}}$ observed in experiments. ${ }^{24}$

\section{B. Effective medium effects}

The percolative nature of the $\mathrm{VO}_{2}$ phase transition allows for an inhomogeneous intermediate state where both metallic and insulating $\mathrm{VO}_{2}$ coexist. The dielectric constants of metal and insulating phase $\mathrm{VO}_{2}$ are distinct; and when both phases can be present in a composite it leads to interesting properties. The average response of the inhomogeneous sample is described by an effective medium, and can have radically different values than either. This leads to quite interesting and novel effects. For example, the inhomogeneity 2 of polycrystalline $\mathrm{VO}_{2}$ mid-transition is responsible for observed memristance ${ }^{19}$ and memory-capacitance ${ }^{15 / 28}$. 


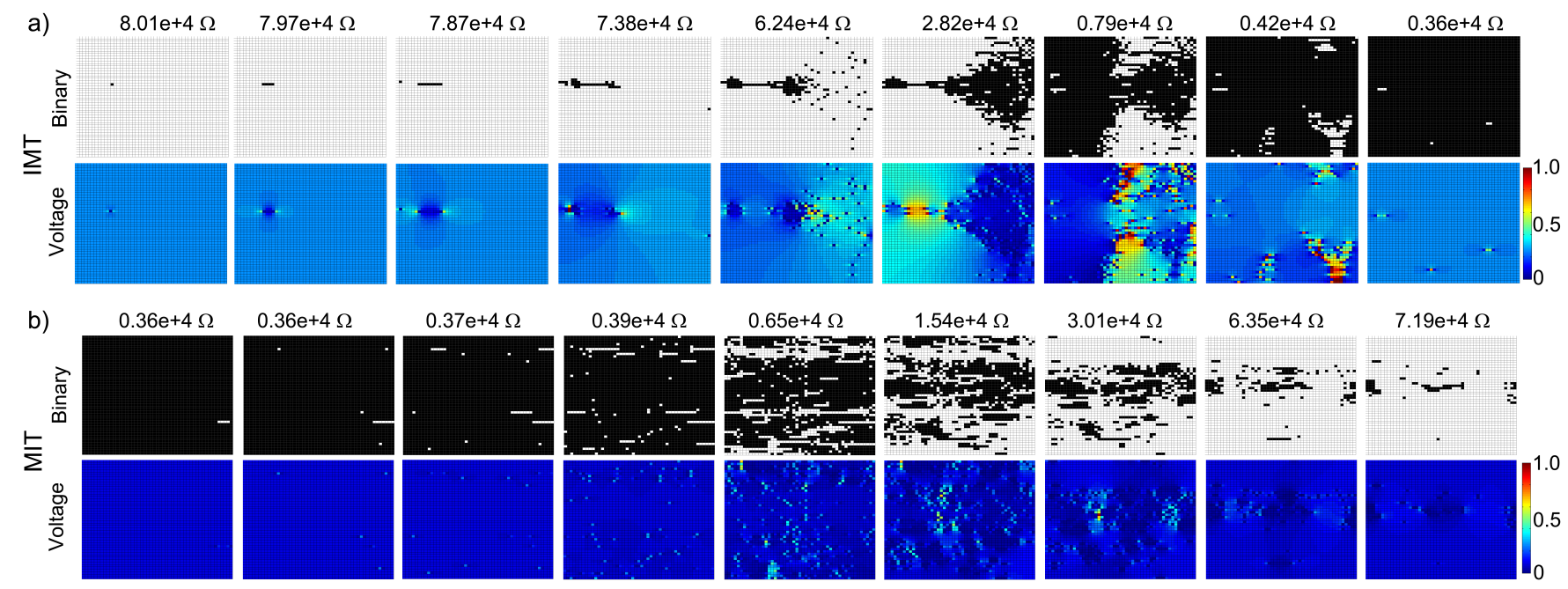

FIG. 8. Step-by-step depiction of the avalanche-like transition for a 50x50 grain network. The time of each frame increases from left to right. a) shows the IMT transition occurring at $V_{D: I M T}$, giving a bi-color plot (top) indicating whether each grain is metallic or insulating and the the voltage (bottom) across each grain in the network. b) shows the same plots for the MIT transition occurring at $V_{D: M I T}$.

The same memory-capacitance as reported in $\frac{15}{15}$ has previously been attributed as playing a key role in voltage-controlled oscillations in $\mathrm{VO}_{2} 24$. This is a question we are situated to investigate in more depth using our model. To look closely at the effects of capacitance on the oscillations, we focus attention on a single IMT transition event. In Figure 9 we plot $\mathrm{C}_{D}(\mathrm{t})$ (as calculated from equations 2 24, along with the familiar $\mathrm{V}_{D}$ and $\mathrm{R}_{D}$.

Looking at Figure 9, as $\mathrm{VO}_{2}$ transitions from insulating to metallic at the IMT, $\mathrm{R}_{D}$ drops monotonically to its metallic-state value. The capacitance $\mathrm{C}_{D}$, however, briefly increases before also decreasing to its metallicstate value. This increase is due to the coexistence of metallic and insulating grains, and is predicted by effective medium (Equation 4). However, as Figure 9 shows, the increase in $\mathrm{C}_{D}$ is a small effect, and is contained to a short timespan near the start of the IMT. This leads us to believe the effective medium behavior of $\mathrm{C}_{D}$ has only a minor influence on the shape of the oscillations, and is not a primary driver. We observe the same increase in $\mathrm{C}_{D}$ at the MIT transition edge, but it also is too small and short-lived an effect to bear responsibility for the oscillations.

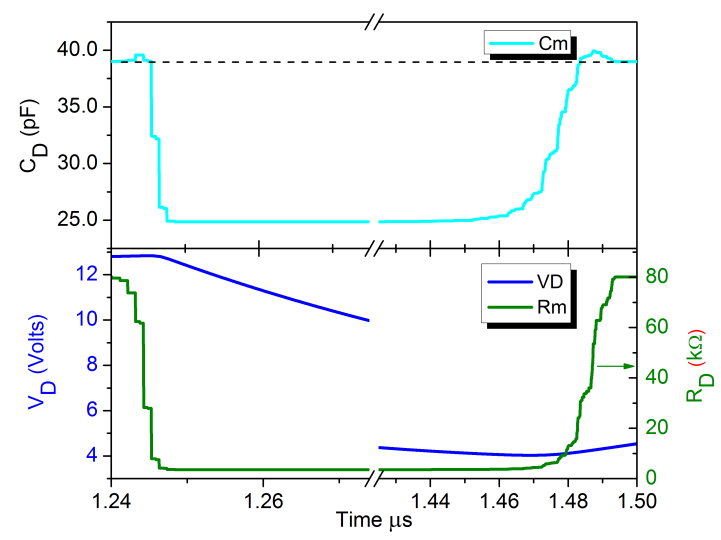

FIG. 9. Model values for $\mathrm{C}_{D}$ plotted with $\mathrm{V}_{D}$ and $\mathrm{R}_{D}$ over one oscillation period. Effective medium within the $\mathrm{VO}_{2}$ (see Equation 2) causes a brief spike in $\mathrm{C}_{D}$ at immediately near the IMT and MIT transitions. As seen, this spike effect is quite small compared to the overall change of both $\mathrm{C}_{D}$ and $\mathrm{R}_{D}$, and exists only for a very brief fraction of the oscillation period. The jagged shape of the model curves for $\mathrm{R}_{D}$ and $\mathrm{C}_{D}$ reveal the small jumps typical of percolation discussed in Section IVA

\section{SUMMARY}

In this work, we have discussed the thermal and electrical driving mechanisms behind observed oscillations occurring in $\mathrm{VO}_{2}$ films. In addition to experimentally confirming the oscillations reported by Y.W. Lee et.al. ${ }^{22}$, we have compiled a numerical model which is able to replicate and explain these oscillations in terms of an voltagetriggered Insulator-to-Metal phase-transition. Tempera- 
ture is known to trigger the IMT in $\mathrm{VO}_{2}$, and temperature plays some role in the shape of the oscillations. However, we find that temperature-only triggering cannot explain oscillations. This result is likely of great importance for applications of these oscillations, as repeated thermalcycling typically appreciably shortens device lifetime.

One question that remains unaddressed is the role, if any, of the structural phase transition in an electric-field triggered transition. The temperature driven IMT in $\mathrm{VO}_{2}$ exhibits a structural transition that happens concurrent with the electronic reconfiguration. However, there is evidence $e^{2 / 6|34| 44}[99$ to suggest the electronic and structural transitions are not necessarily linked, but merely overlaid. This structural electronic decoupling suggests the electronic correlations in $\mathrm{VO}_{2}$ play an important role in the IMT phase transition.

Studies which probe the crystal structure simultaneous with these oscillations have not yet been reported - likely because the time and length scales associated with the $\mathrm{VO}_{2}$ oscillator devices greatly complicate experiments such as x-ray diffraction. However, as mentioned in the introduction (Section II) the question of whether or not the structural transition occurs has great implications about the longevity of these devices. In many applications, devices could easily be expected to perform $10^{12}$ to $10^{14}$ oscillation events over their lifetime, a likely impossibility if crystallographic changes are occurring. An evident goal for the new future is to experimentally investigate the existence of structural transition in oscillations in the near future.

\section{ACKNOWLEDGMENTS}

T.D. acknowledges support from an IC postdoctoral fellowship. M.D. acknowledges partial support from NSF. This research is supported by AFOSR and ETRI

\section{REFERENCES}

${ }^{1} \mathrm{~F}$ J Morin. Oxides which show a metal-to-insulator transition at the neel temperature. Physical Review Letters, 3(1):34-36, 1959. ${ }^{2}$ Mumtaz M. Qazilbash, Markus Brehm, Byung-Gyu Chae, P.-C. Ho, Gregory O. Andreev, Bong-Jun Kim, Sun Jin Yun, A.V. Balatsky, M.B. Maple, Fritz Keilmann, Hyun-Tak Kim, and Dimitri N. Basov. Mott Transition in VO2 revealed by Infrared Spectroscopy and Nano-Imaging. Science, 318(December):1750-1753, 2007.

${ }^{3}$ Mumtaz M. Qazilbash, AA Schafgans, KS Burch, SJ Yun, BG Chae, BJ Kim, Hyun-Tak Kim, and DN Basov. Electrodynamics of the vanadium oxides VO2 and V2O3. Physical Review B, 77(115121), 2008. doi:10.1103/PhysRevB.77.115121 URL http://arxiv.org/abs/0803.2739

${ }^{4}$ M. Liu, B. Pardo, MM Qazilbash, S.J. Yun, BG Chae, BJ Kim, DN Basov, and RD Averitt. Conductivity dynamics in the correlated metallic state of V2O3. In Lasers and Electro-Optics, 2009 and 2009 Conference on Quantum electronics and Laser Science Conference. CLEO/QELS 2009. Conference on, volume 4, pages 1-2. IEEE, 2009. URL http://ieeexplore.ieee.org/xpls/abs_ all.jsp?arnumber $=5225800$
${ }^{5}$ A. Zylbersztejn and N.F. Mott. Metal-insulator transition in vanadium dioxide. Physical Review B, 11(11):4383, 1975.

${ }^{6}$ Hyun-Tak Kim, B G Chae, D H Youn, S L Maeng, G Kim, K Y Kang, and Y S Lim. Mechanism and observation of Mott transition in VO 2 based two- and three-terminal devices. New Journal of Physics, 6:52-70, 2004.

${ }^{7}$ T.M. Rice, H. Launois, and J.P. Pouget. Comment on "VO2: Peierls or Mott-Hubbard? A View from Band Theory". Physical Review Letters, 73(22):9007-9007, 1994.

${ }^{8}$ Renata M Wentzcovitch, Werner W Schulz, and Philip B Allen. VO2: Peierls or Mott-Hubbard? A View from Band Theory. Physical Review Letters, 72(21):3389-3392, 1994.

${ }^{9}$ Andrea Cavalleri, Th. Dekorsy, H. Chong, J. Kieffer, and R. Schoenlein. Evidence for a structurally-driven insulator-tometal transition in VO2: A view from the ultrafast timescale. Physical Review B, 70(16):3-6, October 2004. ISSN 1098-0121. doi:10.1103/PhysRevB.70.161102 URL http://link.aps.org/ doi/10.1103/PhysRevB.70.161102

${ }^{10}$ Andrea Cavalleri, Cs. Tóth, C W Siders, J A Squier, F Ráksi, $\mathrm{P}$ Forget, and $\mathrm{J} \mathrm{C}$ Kieffer. Femtosecond structural dynamics in VO 2 during an ultrafast solid-solid phase transition. $P R L$, 87237401:1-4, 2001.

${ }^{11}$ Matteo Rini, Andrea Cavalleri, Robert W Schoenlein, René López, Leonard C Feldman, Richard F Haglund, Lynn A Boatner, and Tony E Haynes. Photoinduced phase transition in VO 2 nanocrystals : control of surface-plasmon resonance ultrafast. Optics Letters, 30(5):1-3, 2005.

${ }^{12} \mathrm{~S}$ Lysenko, AJ Rua, V Vikhnin, J Jimenez, F Fernandez, and $\mathrm{H}$ Liu. Light-induced ultrafast phase transitions in VO2 thin film. Applied surface science, 252(15):5512-5515, 2006. doi:10.1016/j.apsusc.2005.12.137 URL http://linkinghub. elsevier.com/retrieve/pii/S0169433205018027

${ }^{13}$ Rene Lopez, L. a. Boatner, T. E. Haynes, R. F. Haglund, and L. C. Feldman. Switchable reflectivity on silicon from a composite VO[sub 2]-SiO[sub 2] protecting layer. Applied Physics Letters, 85(8):1410, 2004. ISSN 00036951. doi:10.1063/1.1784546 URL http://link.aip.org/link/APPLAB/v85/i8/p1410/s1\&Agg=doi

${ }^{14}$ Tom Driscoll, S Palit, Mumtaz M. Qazilbash, Markus Brehm, Fritz Keilmann, Byung-Gyu Chae, Sun-Jin Yun, Hyun-Tak Kim, Nan Marie Jokerst, David R. Smith, and Dimitri N. Basov. Dynamic tuning of an infrared hybrid-metamaterial resonance using vanadium dioxide. Applied Physics Letters, 93(024101), 2008. doi:10.1063/1.2956675. URL http://ieeexplore.ieee. org/xpls/abs_all.jsp?arnumber $=4838777$

${ }^{15}$ Tom Driscoll, Hyun-Tak Kim, Byung-Gyu Chae, Bong-Jun Kim, Yong-Wook Lee, Nan Marie Jokerst, S Palit, David R. Smith, Massimiliano Di Ventra, and Dimitri N. Basov. Memory metamaterials. Science (New York, N.Y.), 325(5947):1518-21, September 2009. ISSN 1095-9203. doi:10.1126/science.1176580 URL http://www.ncbi.nlm.nih.gov/pubmed/19696311.

${ }^{16}$ Matthew J Dicken, Koray Aydin, Imogen M Pryce, Luke A Sweatlock, M Boyd, Sameer Walavalkar, James Ma, and Harry A Atwater. Frequency tunable near-infrared metamaterials based on VO 2 phase transition. Optics Express, 17(20):295-298, 2009.

${ }^{17}$ B.J. Kim, Y.W. Lee, B.G. Chae, S.J. Yun, S.Y. Oh, Y.S. Lim, and Hyun-Tak Kim. Temperature dependence of Mott transition in $\mathrm{VO} \backslash \_2$ and programmable critical temperature sensor. Arxiv preprint cond-mat/0609033, 009033v1, 2006. URL http://arxiv.org/abs/cond-mat/0609033

${ }^{18}$ Tom Driscoll, J. Quinn, S. Klein, Hyun-Tak Kim, B. J. Kim, Yuriy V. Pershin, Massimiliano Di Ventra, and D. N. Basov. Memristive adaptive filters. Applied Physics Letters, 97(9):093502, 2010. ISSN 00036951. doi: 10.1063/1.3485060 URL http://link.aip.org/link/APPLAB/ v97/i9/p093502/s1\&Agg=doi

${ }^{19}$ Tom Driscoll, Hyun-Tak Kim, B.G. Chae, Massimiliano Di Ventra, and DN Basov. Phase-transition driven memristive system. Applied Physics Letters, 95(4):043503, 2009. URL http: //link.aip.org/link/?APPLAB/95/043503/1 
${ }^{20}$ YV Pershin. Memory effects in complex materials and nanoscale systems. Advances in Physics, 00(00):1-71, 2011. doi: 10.1080/0001873YYxxxxxxxx URL http://www.tandfonline. com/doi/abs/10.1080/00018732.2010.544961

${ }^{21}$ Aurelian Crunteanu, Julien Givernaud, Jonathan Leroy, David Mardivirin, Corinne Champeaux, Jean-Christophe Orlianges, Alain Catherinot, and Pierre Blondy. Voltage- and currentactivated metalinsulator transition in VO 2 -based electrical switches: a lifetime operation analysis. Science and Technology of Advanced Materials, 11(6):065002, December 2010. ISSN 1468-6996. doi:10.1088/1468-6996/11/6/065002 URL http://stacks.iop.org/1468-6996/11/i=6/a=065002?key= crossref.3559a62a92655ee932bf6fc313bbb47a

${ }^{22}$ Y.W. Lee, B.J. Kim, J.W. Lim, S.J. Yun, Sungyoul Choi, B.G. Chae, G. Kim, and Hyun-Tak Kim. Metal-insulator transition-induced electrical oscillation in vanadium dioxide thin film. Applied Physics Letters, 92(16):162903, $2008 . \quad$ doi: 10.1063/1.2911745 URL http://link.aip.org/link/?APPLAB/ $92 / 162903 / 1$

${ }^{23}$ Joe Sakai. High-efficiency voltage oscillation in VO[sub 2] planer-type junctions with infinite negative differential resistance. Journal of Applied Physics, 103(10):103708, 2008. ISSN 00218979. doi:10.1063/1.2930959 URL http://link.aip.org/ link/JAPIAU/v103/i10/p103708/s1\&Agg=doi

${ }^{24}$ Hyun-Tak Kim, Bong-Jun Kim, Sungyoul Choi, Byung-Gyu Chae, Yong Wook Lee, Tom Driscoll, Mumtaz M. Qazilbash, and D. N. Basov. Electrical oscillations induced by the metal-insulator transition in $\mathrm{VO}[\mathrm{sub} 2]$. Journal of $A p$ plied Physics, 107(2):023702, 2010. ISSN 00218979. doi: 10.1063/1.3275575 URL http://link.aip.org/link/JAPIAU/ $\mathrm{v} 107 / \mathrm{i} 2 / \mathrm{p} 023702 / \mathrm{s} 1 \&$ Agg=doi

${ }^{25}$ B.J. Kim, Giwan Seo, Y.W. Lee, Sungyoul Choi, and HyunTak Kim. Linear Characteristics of a MetalInsulator Transition Voltage and Oscillation Frequency in VO2 Devices. Electron Device Letters, IEEE, 31(11):1314-1316, 2010. URL http: //ieeexplore.ieee.org/xpls/abs_all.jsp?arnumber=5595090

${ }^{26}$ Amos Sharoni, J.G. Ram\'\irez, and I.K. Schuller. Multiple avalanches across the metal-insulator transition of vanadium oxide nanoscaled junctions. Physical review letters, 101 (2):26404, 2008. doi:10.1103/PhysRevLett.101.026404 URL http://link.aps.org/doi/10.1103/PhysRevLett.101.026404

${ }^{27}$ J.G. Ram〉'\irez, Amos Sharoni, Y Dubi, ME Gomez, and I.K. Schuller. First-order reversal curve measurements of the metal-insulator transition in $\mathrm{VO}_{2}$ : Signatures of persistent metallic domains. Physical Review B, 79(23):235110, 2009. doi:10.1103/PhysRevB.79.235110 URL http://prb.aps.org/ abstract/PRB/v79/i23/e235110

${ }^{28}$ Massimiliano Di Ventra, Yuriy V. Pershin, and Leon O. Chua. Circuit elements with memory: memristors, memcapacitors and meminductors. Proceedings of the IEEE, 97(8):1371-1372, January 2009. doi:10.1109/JPROC.2009.2021077 URL http:// arxiv.org/abs/0901.3682

${ }^{29}$ John Rozen, Rene Lopez, Richard F. Haglund, and Leonard C. Feldman. Two-dimensional current percolation in nanocrystalline vanadium dioxide films. Applied Physics Letters, 88(8):081902, 2006. ISSN 00036951. doi: 10.1063/1.2175490 URL http://link.aip.org/link/APPLAB/ $\mathrm{v} 88 / \mathrm{i} 8 / \mathrm{p} 081902 / \mathrm{s} 1 \&$ Agg $=$ doi

${ }^{30}$ Jun Dai, Xingzhi Wang, Ying Huang, and Xinjian Yi. Modeling of temperature-dependent resistance in micro- and nanopolycrystalline $\mathrm{VO}[\mathrm{sub} 2]$ thin films with random resistor networks. Optical Engineering, 47(3):033801, 2008. ISSN 00913286. doi: 10.1117/1.2894146 URL http://link.aip.org/link/OPEGAR/ $\mathrm{v} 47 / \mathrm{i} 3 / \mathrm{p} 033801 / \mathrm{s} 1 \& \mathrm{Agg}=\mathrm{doi}$

${ }^{31}$ Mei Pan, Hongmei Zhong, Shaowei Wang, Jie Liu, Zhifeng Li, Xiaoshuang Chen, and Wei Lu. Properties of VO 2 thin film prepared with precursor VO ( acac ) 2. Journal of Crystal Growth, 265:121-126, 2004. doi:10.1016/j.jcrysgro.2003.12.065

${ }^{32}$ N. R. Mlyuka and R. T. Kivaisi. Correlation between optical, electrical and structural properties of vanadium diox- ide thin films. Journal of Materials Science, 41(17):56195624, June 2006. ISSN 0022-2461. doi:10.1007/s10853-0060261-y URL http://www.springerlink.com/index/10.1007/ s10853-006-0261-y

${ }^{33}$ Jeehoon Kim, Changhyun Ko, Alex Frenzel, Shriram Ramanathan, and Jennifer E. Hoffman. Nanoscale imaging and control of resistance switching in $\mathrm{VO}[\mathrm{sub} 2]$ at room temperature. Applied Physics Letters, 96(21):213106, 2010. ISSN 00036951. doi:10.1063/1.3435466 URL http://link.aip.org/ link/APPLAB/v96/i21/p213106/s1\&Agg=doi

${ }^{34}$ Mumtaz M. Qazilbash, a. Tripathi, a. Schafgans, Bong-Jun Kim, Hyun-Tak Kim, Zhonghou Cai, M. Holt, J. Maser, F. Keilmann, O. Shpyrko, and D. Basov. Nanoscale imaging of the electronic and structural transitions in vanadium dioxide. Physical Review B, 83(16):1-7, April 2011. ISSN 1098-0121. doi: 10.1103/PhysRevB.83.165108 URL http://link.aps.org/doi/ 10.1103/PhysRevB.83.165108

${ }^{35}$ Alex. Frenzel, Mumtaz M. Qazilbash, M. Brehm, Byung-Gyu Chae, Bong-Jun Kim, Hyun-Tak Kim, A. Balatsky, F. Keilmann, and D. Basov. Inhomogeneous electronic state near the insulator-to-metal transition in the correlated oxide VO2. Physical Review B, 80(11):1-7, September 2009. ISSN 1098-0121. doi:10.1103/PhysRevB.80.115115 URL http://link.aps.org/ doi/10.1103/PhysRevB.80.115115

${ }^{36}$ N. a. Poklonski, a. a. Kocherzhenko, a. I. Benediktovitch, V. V. Mitsianok, and a. M. Zaitsev. Simulation of dc conductance of two-dimensional heterogeneous system: application to carbon wires made by ion irradiation on polycrystalline diamond. Physica Status Solidi (B), 243(6):1212-1218, May 2006. ISSN 03701972. doi:10.1002/pssb.200541079 URL http://doi.wiley.com/ $10.1002 / \mathrm{pssb} .200541079$

${ }^{37}$ Dmitry Ruzmetov, Gokul Gopalakrishnan, Jiangdong Deng, V. Narayanamurti, and Shriram Ramanathan. Electrical triggering of metal-insulator transition in nanoscale vanadium oxide junctions. Journal of Applied Physics, 106(8):083702-083702, 2009. doi:10.1063/1.3245338. URL http://ieeexplore.ieee. org/xpls/abs_all.jsp?arnumber $=5292009$

${ }^{38}$ G. Stefanovich, A Pergament, and D Stefanovich. Electrical switching and Mott transition in VO2. Journal of Physics: Condensed Matter, 12:8837, 2000. URL http://iopscience.iop. org/0953-8984/12/41/310

${ }^{39}$ Gokul Gopalakrishnan, D. Ruzmetov, and Shriram Ramanathan. On the triggering mechanism for the metalinsulator transition in thin film VO 2 devices: electric field versus thermal effects. Journal of materials science, 44(19):5345-5353, 2009. doi: 10.1007/s10853-009-3442-7. URL http://www.springerlink. com/index/7U24554G53291604.pdf

${ }^{40}$ Massimiliano Di Ventra. Electrical transport in nanoscale systems. Cambridge University Press, Cambridge, 2008.

${ }^{41}$ Feliks Chudnovskiy, Serge Luryi, and Boris Spivak. Switching device based on first-order metal- insulator transition induced by external electric field. Future Trends in Microelectronics: the Nano Millennium, pages 148-155, 2002.

${ }^{42}$ Mumtaz M. Qazilbash, Z. Q. Li, V. Podzorov, M. Brehm, F. Keilmann, B. G. Chae, Hyun-Tak Kim, and D. N. Basov. Electrostatic modification of infrared response in gated structures based on VO[sub 2]. Applied Physics Letters, 92(24):241906, 2008. ISSN 00036951. doi:10.1063/1.2939434 URL http: //link.aip.org/link/APPLAB/v92/i24/p241906/s1\&Agg=doi

${ }^{43}$ Tai-Lung Wu, Luisa Whittaker, Sarbajit Banerjee, and G. Sambandamurthy. Temperature and voltage driven tunable metalinsulator transition in individual $\mathrm{W}_{x} \mathrm{~V}_{1-x} \mathrm{O}_{2}$ nanowires. Physical Review B, 83(7):2-5, February 2011. ISSN 1098-0121. doi: 10.1103/PhysRevB.83.073101 URL http://link.aps.org/doi/ 10.1103/PhysRevB.83.073101

${ }^{44}$ E. Arcangeletti, L. Baldassarre, D. Di Castro, S. Lupi, L. Malavasi, C. Marini, a. Perucchi, and P. Postorino. Evidence of a Pressure-Induced Metallization Process in Monoclinic VO2. Physical Review Letters, 98(19):1-4, May 2007. ISSN 0031-9007. doi:10.1103/PhysRevLett.98.196406 URL 
http://link.aps.org/doi/10.1103/PhysRevLett.98.196406

${ }^{45}$ Jiang Wei, Zenghui Wang, Wei Chen, and D.H. Cobden. New aspects of the metalinsulator transition in single-domain vanadium dioxide nanobeams. Nature Nanotechnology, 4(7):420-424, 2009. doi:10.1038/NNANO.2009.141 URL http://www.nature. com/nnano/journal/v4/n7/abs/nnano.2009.141.html

${ }^{46}$ Tao Yao, Xiaodong Zhang, Zhihu Sun, Shoujie Liu, Yuanyuan Huang, Yi Xie, Changzheng Wu, Xun Yuan, Wenqing Zhang, Ziyu Wu, Guoqiang Pan, Fengchun $\mathrm{Hu}$, Lihui Wu, Qinghua Liu, and Shiqiang Wei. Understanding the Nature of the Kinetic Process in a VO2 Metal-Insulator Transition. Physical Review Letters, 105(22):2-5, November 2010. ISSN 0031-9007. doi: 10.1103/PhysRevLett.105.226405 URL http://link.aps.org/ doi/10.1103/PhysRevLett.105.226405

${ }^{47}$ Bong-Jun Kim, Yong Wook Lee, Byung-Gyu Chae, Sun Jin Yun, Soo-Young Oh, Hyun-Tak Kim, and Yong-Sik Lim. Temperature dependence of the first-order metal-insulator transition in $\mathrm{VO}[$ sub 2] and programmable critical temperature sensor. $A p$ - plied Physics Letters, 90(2):023515, 2007. ISSN 00036951. doi: 10.1063/1.2431456 URL http://link.aip.org/link/APPLAB/ v90/i2/p023515/s1\&Agg=doi

${ }^{48}$ Bong-Jun Kim, Yong Lee, Sungyeoul Choi, Jung-Wook Lim, Sun Yun, Hyun-Tak Kim, Tae-Ju Shin, and Hwa-Sick Yun. Micrometer x-ray diffraction study of VO2 films: Separation between metal-insulator transition and structural phase transition. Physical Review B, 77(23):1-5, June 2008. ISSN 1098-0121. doi: 10.1103/PhysRevB.77.235401 URL http://link.aps.org/doi/ 10.1103/PhysRevB.77.235401.

${ }^{49}$ Hyun-Tak Kim, Byung-Gyu Chae, Doo-Hyeb Youn, Gyungock Kim, Kwang-Yong Kang, Seung-Joon Lee, Kwan Kim, and Yong-Sik Lim. Raman study of electric-field-induced first-order metal-insulator transition in $\mathrm{VO}[\mathrm{sub} 2]$-based devices. Applied Physics Letters, 86(24):242101, 2005. ISSN 00036951. doi: 10.1063/1.1941478 URL http://link.aip.org/link/APPLAB/ v86/i24/p242101/s1\&Agg=doi 